\title{
DEVELOPMENT OF A MODEL-BASED DIGITAL AND VISUAL WHEAT GROWTH SYSTEM
}

\author{
Liang Tang, Hui Liu, Yan Zhu, Weixing Cao* \\ High-Tech Key Laboratory of Information Agriculture of Jiangsu Province, Nanjing \\ Agricultural University, Nanjing, 210095, China \\ "Corresponding author: Tel: +86-25-84395845, Fax: 86-25-84396565, Email: Caow@njau.edu.cn
}

Abstract: Driven by soil, variety, weather and management databases and integrating process-based growth simulation model, morphological model and visualization model, a model-based digital and visual wheat growth system (MDVWGS) was developed using component-based software and visualization techniques. The system was programmed by the .Net framework with the language of C\# and CsGL Library was used for realizing 2D and 3D graphics application and visualization. The implemented system could be used for predicting growth processes and visualizing morphological architecture of wheat plant under various environments, genotypes and management strategies, and has the functions as data management, dynamic simulation, strategy evaluation, real-time prediction, temporal and spatial analysis, visualization output, expert consultation and system help. The MDVWGS should be useful for construction and application of digital farming system and provide a precise and scientific tool for cultivar design, cultural regulation and productivity evaluation under different growing conditions.

Keywords: Wheat; Growth model; Morphological model; Functional-structural plant model; Visualization

\section{INTRODUCTION}

Crop modeling based on ecophysiological processes of plant growth and development has become an important field of research during the past decades. The main emphasis in crop growth modeling has been put on developing models by description of fundamental biophysical, biochemical

Tang, L., Liu, H., Zhu, Y. and Cao, W., 2008, in IFIP International Federation for Information Processing, Volume 259; Computer and Computing Technologies in Agriculture, Vol. 2; Daoliang Li; (Boston: Springer), pp. 1113-1120. 
and physiological processes of growth and yield formation (Bouman et al., 1996; van Ittersum et al., 2003). This type of models is commonly referred to as process-based models, while plant architecture is normally addressed in a simplified manner. In recent years, approaches have been developed to describe the geometric structure of plant. L-system or similar approaches were used to simulate the architecture of plants (de Reffye et al., 1988; Prusinkiewicz and Lindenmayer, 1990), and these models gained in versatility and provided with graphical capability.

A new modeling approach named functional-structural plant model (FSPM) concerned with integration of architecture and resource allocation as aspects of plant function was developed in the mid 1990s (Godin, 2000; Sievanen et al., 2000). For agricultural crops several reports have been published during the last years dealing with FSPM under development (Drouet and Pages, 2003; Fournier et al., 2003; Hanan et al., 2003; Yan et al., 2004). This type of model incorporates the physiological processes into the architecture model, aiming at 3D geometric structure of plant become mechanism and process-based. But most of these models link an architecture model with a canonical mathematical model (Renton et al., 2005), thus these models can simulate only a few ecophysiological processes.

Many crop growth model-based decision support systems have been established, such as APSIM (McCown et al., 1996), DSSAT (Jones et al., 2003) and GMDSSWM (Pan, 2005), which can simulate crop growth and development with different management strategies and making reasonable decisions about crop management based on the results of crop growth simulation. On the other hand, plant architecture software as AMAP (Jaeger M and de Reffye P, 1992; Godin et al., 1997) and vlab (Federl and Prusinkiewicz, 1999) integrating computer graphics aim at plant architecture modeling and visualization. So far both crop simulation and decision support and architecture visualization remain to be incorporated into one system or software for a comprehensive representation of crop growth system.

The present study developed a digital and visual wheat growth system in order to gather different eco-physiological modules and visualization techniques for (i) simulating eco-physiological processes as development, growth, yield and quality and making reasonable decisions about crop management (ii) simulating topological and 3D structure of wheat at organ, individual, population levels; (iii) demonstrating the real-time visualization of wheat growth responses to different growth conditions and varieties.

We hereafter achieved the above objectives by integrating a processbased comprehensive growth simulation model WheatGrow (Yan, 1999; Liu, 2000; Pan, 2005), architecture model WheatArch (Cheng, 2004, Tan, 
2006) and visualization model for producing a new functional-structural model WheatFM. This included linking the functional processes of WheatGrow with dynamic structures of WheatArch, as well as integrating component-based software and computer graphic technology for visualization.

\section{OVERALL STRUCTURE OF MDVWGS}

The MDVWGS could simulate growth, architecture, yield and quality of wheat growing on a uniform area of land under prescribed or simulated management as well as under the changed soil water and nitrogen conditions. It is comprised of database, models, applications and interface (Fig. 1).

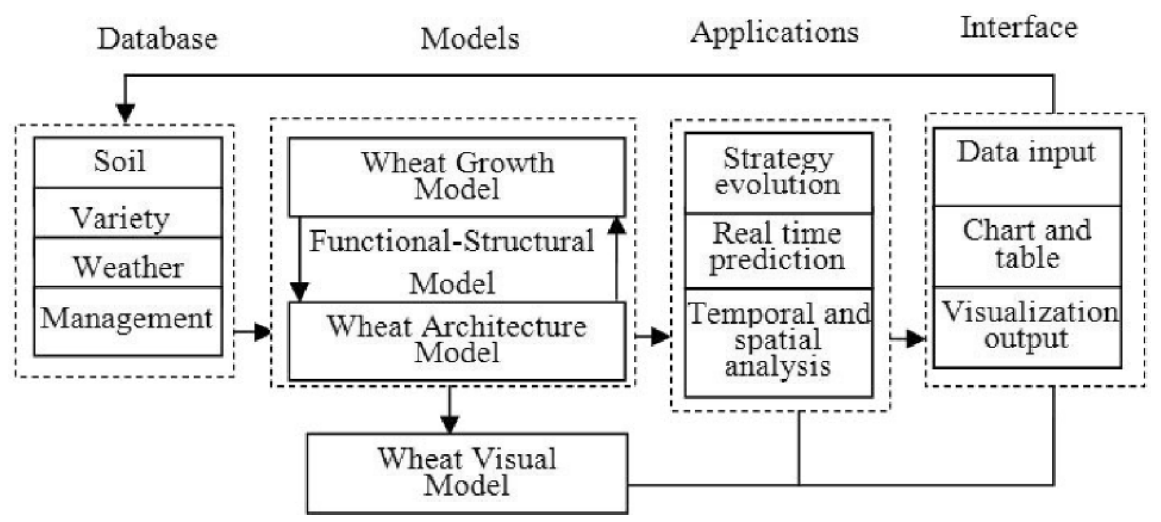

Fig. 1. Structural framework of MDVWGS

\subsection{Database}

The database includes weather, soil, variety and management data. The weather data has daily records of date, maximum and minimum air temperature, sunshining hours and rainfall. The soil data has typical soil parameters for soil module, e.g. soil water content, ammonium $\mathrm{N}$ and nitrate in soil layers, thickness of a layer, actual number of soil layers. The variety data includes genotype-specific parameters, e.g. variety name, phenological parameters, thousand-seed weight and parameters of architecture model. Management data contains some common practices of wheat management. 


\subsection{Models}

The main models include growth simulation model WheatGrow, the architecture formation model WheatArch, visualizaton model, and module for model integration.

\subsubsection{Growth simulation model WheatGrow}

WheatGrow is a field scale, weather-driven, process-based dynamic simulation model (Yan, 1999; Liu, 2000; Pan, 2005), which operates with a daily time-step, including 6 submodels for simulating phasic and phenological development, morphological and organ formation, photosynthesis and dry matter accumulation, yield and quality formation, soil water relation soil water relation and dynamic nutrient $(\mathrm{N}, \mathrm{P}, \mathrm{K})$ balance.

\subsubsection{The architecture model WheatArch}

The architecture model WheatArch allows dynamic construction of geometric structure of actual wheat plant, which can simulate the geometrical and topological structure at organs, individual and population levels in relation to growing degree days (GDD), including topological structure, leaf morphology (leaf length, width, angle and curvature), sheath and internode (width, length), spike (length, width) sub-models and threedimensional structural model (Cheng, 2004, Tan, 2006).

\subsubsection{Integration of WheatGrow and WheatArch}

The WheatGrow runs continuously and independently driven by data of weather, variety, soil and management, simulating the functional processes of wheat and providing basic input for WheatArch model. The model WheatGrow was used to calculate: (1) phasic and phenological development to control the runtime of WheatArch from emergence to maturity; (2) characters of population architecture; (3) assimilate accumulation and partitioning, e.g. WheatGrow provides leaf area and leaf weight, while WheatArch simulates individual leaf initiation and growth, and both would be linked to build process-based leaf morphological model (4) water and nutrient factors for quantifying the limiting factors of water and NPK nutrients for WheatArch. These were taken as the inputs of WheatArch. 


\subsubsection{Visualization model}

Visualization model includes geometry, texture, illumination submodels. Geometric submodel includes simulation of several organs, using different geometric shapes (e.g. cylinder) to model leaf, stem and spike; the texture model is constructed by using the photos of organs at different stages with reality as textures and integrated with geometric model; illumination model in OPGL is adopted for simulating the illumination effect in the reality.

\subsection{Applications}

Different types of applications were accomplished in MDVWGS by using different functions on a daily basis, such as temporal and spatial analysis, strategy evaluation and real time prediction.

\section{$2.4 \quad$ Interface}

The interface of MDVWGS included the initial data input, digital and visualization output. The results of simulation strategy in digits can be represented by chart and table, and the results also can be displayed by visualization through the computer graphic technology, including scene controlling as zoom in or zoom out, changing the point of view.

\section{FUNCTION DESCRIPTIONS}

In MDVWGS, Multiple functions as simulating time-course processes of growth and development, architecture, yield and quality formation, soil water and nutrient dynamics under various environmental conditions, production levels and genetic parameters were developed so that the system could realize the functions of dynamic simulation, strategy evaluation and real time forecasting which have been implemented by Pan (2005) (Fig. 2). Result of these functions can be displayed by digits and visualized as computer images.

\section{SYSTEM IMPLEMENTATION}

The system was operated under windows 2003 server on PC with $1 \mathrm{G}$ of RAM and AMD althon 2500+. The MDVWGS applied Access 2003 to designing database, $\mathrm{C} \#$ in the framework of .NET to programming the 


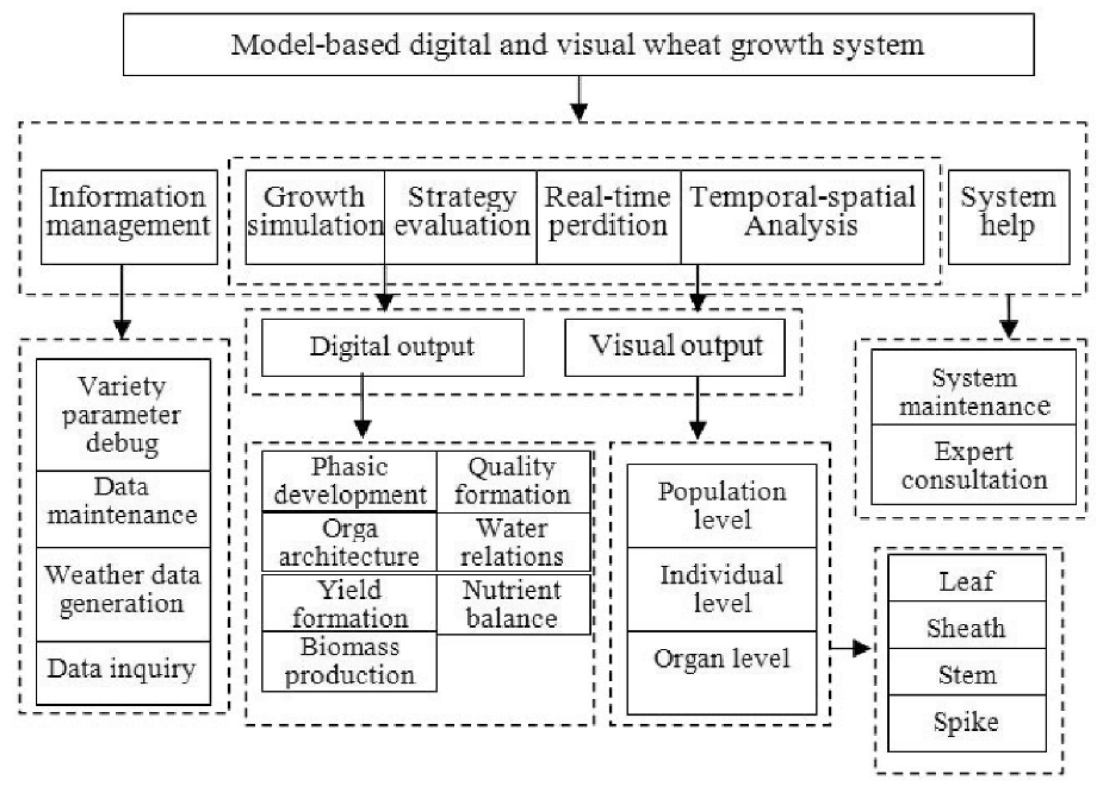

Fig. 2. Functions of MDVWGS

interface and programming growth model components based on the COM standard. Visualization model is designed by CSGL (C sharp Graphics Library) which implements a wrapper for the powerful C-library OpenGL allowing use of any .NET language.

\subsection{System display}

Part of system application is presented as follows, including the interface of MDVWGS for main menu (Fig. $3 \mathrm{a}$ ), digital results displayed by table (Fig. 3b), the visual output of dynamic individual plant growth (Fig. 3c, d).

\section{DISCUSSIONS}

Based on a process-based growth simulation model WheatGrow, architecture model WheatAM and visualization model, a digital and visual wheat growth system (MDVWGS) was developed using component-based software and visualization techniques, which enable ecophysiologists to investigate the interaction of organs, single plants or plant stands with their biotic and abiotic environment in a unique way. The implemented system integrated prediction function and decision support function, can simulate eco-physiological processes as development, growth, yield and quality, the 


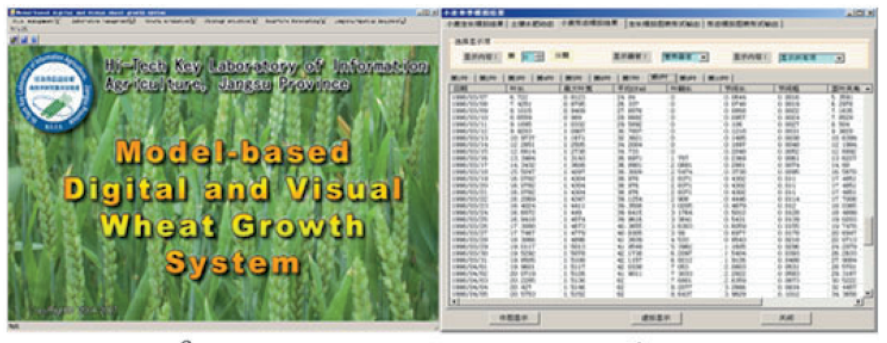

a

b

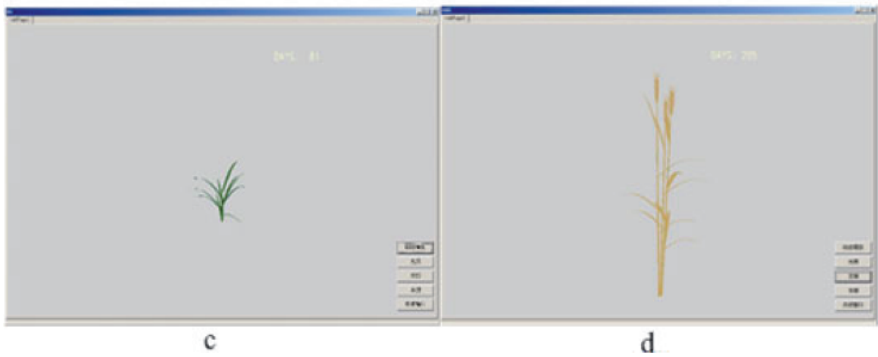

Fig. 3. System exhibition of interface for main menu (a), table output (b), visual output (c, d)

structure of wheat dynamics in mechanism and make decisions for mamagement, demonstrate the visualization of wheat growth under various environments, genotypes and management strategies, which providing a precise and scientific tool for cultivar design, cultural regulation and productivity evaluation under different growing conditions.

Systems like MDVWGS would be put into potential for applications of this technology in research, decision support, education and extension (Room, et al., 1996). In order to make the system more applicable and stability, the next steps will be: (1) to incorporate more physiological processes in the model to enhance the application of the model, e.g. calculation of radiation interception and inter-plant competition which would be feedback to growth model; (2) to extend the variety, soil and weather database, so that the system could be used in further comprehensive conditions; (3) to incorporate the root architecture model and visualization into the MDVWGS; (4) to link the MDVWGS with GIS, RS and management knowledge model for using in precision and digital farm.

\section{ACKNOWLEDGEMENTS}

We acknowledge the financial support from the State Hi-tech R\&D Plan of China (2006AA10Z219) and the Hi-tech R\&D Plan of Jiangsu Province (BG2004320). 


\section{REFERENCES}

Bouman B A, Van K H, Van L H, Rabbinge R. The'School of de Wit'Crop Growth Simulation Models: A pedigree and Historical Overview. Agricultural Systems, 1996, 52(2): 171-198.

Cheng G Q. Studies on Simulation and Visualization of Morphogenesis in Wheat. Taian: Shangdong Agricultural University, 2004. (in Chinese)

de Reffye P, Edelin C, Fran J, Jaeger M, Puech C. Plant models faithful to botanical structure and development. Computer Graphics, 1988, 22(4): 151-158.

Drouet J L, Pages L. GRAAL: A model of GRowth, Architecture and carbon ALlocation during the vegetative phase of the whole maize plant. Model description and parameterisation. Ecological Modelling, 2003, 165(2): 147-173.

Federl P, Prusinkiewicz P. Virtual laboratory: An interactive software environment for computer graphics. Computer Graphics International, Proceedings, 1999, 93-100.

Fournier C, Andrieu B. ADEL-Wheat: A 3D architectural model of wheat development plant growth modeling and applications. Plant growth modeling and applications: proceedingsPMA03: 2003 international symposium on plant growth modeling, simulation, visualization and their applications, Beijing, China, October, 2003, 13-16.

Godin C, Costes E, Caraglio Y. Exploring plant topological structure with the AMAPmod software: An outline. Silva Fennica, 1997, 31: 3, 357-368.

Godin C. Representing and encoding plant architecture: A review. Ann. For. Sci. 2000, 57: 413-438.

Hanan J S, Hearn A B. Linking physiological and architectural models of cotton. Agricultural Systems, 2003, 75(1): 47-77.

Jaeger M, De R P. Basic concepts of computer simulation of plant growth. Journal of biosciences, 1992, 17(3): 275-291.

Jones J W, Hoogenboom G, H., Porter C, Boote K J, Batchelor W D, Hunt L A, Wilkens P W, Singh U, Gijsman A J, Ritchie J T. The DSSAT cropping system model. European Journal of Agronomy, 2003, 18: 235-265.

Liu T M. Simulation on photosynthetic production and dry matter partitioning in wheat. Nanjing: Nanjing Agricultural University, 2001. (in Chinese)

McCown R L, Hammer G L, Hargreaves J N G, Holzworth D P, Freebairn D M. APSIM: A Novel Software System for Model Development, Model Testing and Simulation in Agricultural Systems Research. Agricultural Systems, 1996, 50: 255-271.

Pan J. Study on wheat growth simulation and decision support system. Nanjing: Nanjing Agricultural University, 2005. (in Chinese)

Prusinkiewicz P, Lindenmayer A. The algorithmic beauty of plants. Springer-Verlag New York, Inc. New York, NY, USA, 1990.

Renton M, Kaitaniemib P, Hanana J. Functional-structural plant modelling using a combination of architectural analysis, L-systems and a canonical model of function, Ecological Modelling, 2005, pp. 277-298.

Room P M, Hanan J S, Prusinkiewicz P. Virtual plants: New perspectives for ecologists, pathologists and agricultural scientists. Trends in Plant Science, 1996, 1(1): 33-38.

Sievanen R, Nikinmaa E, Nygren P, Ozier-lafontaine H, Perttunen J, Hakula H. Components of functional-structural tree models. Ann. For. Sci., 2000, 57: 399-412.

Tan Z H. Studies on simulation model of morphological development in wheat plant. Nanjing, 2006. (in Chinese)

Van Ittersum M, Leffelaar P A, Van K H, Kropff M J, Bastiaans L, Goudriaan J. On approaches and applications of the Wageningen crop models. European Journal of Agronomy, 2003, 18(3): 201-234.

Yan H P, Kang M Z, de Reffye P, Dingkuhn M. A dynamic, architectural plant model simulating resource-dependent growth. Annals of botany, 2004, 93(5): 591-602.

Yan M C. A process-based mechanistic model for phasic and phonological development and organ formation in wheat. Nanjing: Nanjing Agricultural University, 1999. (in Chinese) 